\title{
Practical Issues in the Decision Making and Management of Acute lower Gastrointestinal Bleeding: An Algorithmic Approach
}

\author{
Dr Deeplaxmi Borle, Dr Senthil Kumar MP
}

\begin{abstract}
:
Acute lower Gastro intestinal bleeding is a relatively common problem in the emergency department. Etiology is diverse as is the degree of bleeding and natural history. Hence there are a number of subtle aspects of clinical assessment, investigations and management which require attention and application fr optimal outcomes. The goal of this review is to highlight the practical issues in managing the condition from the perspective of a medical officer or a junior resident in surgery who is on-call in the emergency department. A working algorithm incorporating the key management issues is also presented.
\end{abstract}

\section{INTRODUCTION}

Lower GI bleeding is defined as bleeding which originates distal to the ligament of Trietz. This usually presents as passage of bright red or dark red blood per rectum. Rectal bleeding is a relatively common problem requiring emergency admission. However, severe bleeding with significant haemodynamic compromise needing urgent intervention is uncommon. In about $80-85 \%$ the bleeding stops spontaneously. Overall mortality rates for lower GI bleeding are typically < 5\% (Longstreth 1997, Bramley 1996, Peura 1997, Kolef 1995). However, in patients who develop lower GI bleed after admission to the hospital for some other condition have a higher mortality rate of up o $23 \%$ (Longstreth 1997).

In patients with significant hematochezia, colorectal causes account for 70-80\%, an upper GI source is the cause in 10-15\% while the small bowel is the cause in 0.7- 25\% (Farrell 2005; Hoedema 2005). Common anorectal problems are haemorrhoids, fissure, proctitis, cancer and trauma. Common colonic causes are diverticular disease, colitis (inflammatory bowel disease, infective colitis, ischaemic and radiation colitis), neoplasia and angiodysplasia.

Table1. Causes at different anatomical levels

\begin{tabular}{|l|l|l|l|}
\hline \multicolumn{1}{|c|}{ Anorectal } & \multicolumn{1}{|c|}{ Colonic } & \multicolumn{1}{c|}{ Small bowel } & \multicolumn{1}{c|}{ Other } \\
\hline Haemorrhoids and rectal & Diverticular disease & Meckel's diverticulum & UGI haemorrhage \\
varices & Angiodysplasia & Jejunal diverticulum & $\begin{array}{l}\text { presenting as LGI } \\
\text { bleed }\end{array}$ \\
Fissure & Inflammatory bowel disease & Aortoenteric fistula & \\
Neoplasia & Neoplasia & & \\
Inflammatory bowel disease & Infective colitis & & \\
Radiation proctitis & Ischaemic colitis & & \\
Angiodysplasia & Ulcers & & \\
Solitary Ulcer & Neutropenic colitis & & \\
Trauma & & & \\
\hline
\end{tabular}

\section{MANAGEMENT OF LOWER GI BLEED}

\section{(A) General Principles}

- All patients presenting with a history of lower GI bleed, seen in the accident and emergency department, should be discussed with the middle grade surgeon on-call as soon as possible, if they are seen initially by a junior member of the on-call team.

There are a few key questions that need to be addressed by the attending surgical team, which will guide the diagnostic and therapeutic path that is appropriate for that particular patient.

These are:

1. How severe is the bleeding? 
Deeplaxmi Borle \& Senthil Kumar, "Practical Issues in the Decision Making and Management of Acute lower Gastrointestinal Bleeding: an Algorithmic Approach"

2. In what context is it occurring? (Co-morbidity of the patient; available resources on that day - eg endoscopy services, access to interventional radiology, high dependency bed availability)

3. Where is the bleeding from? (upper GI or Lower GI)

4. What is the likely lesion?

These will help decide:

1. Whether the patient needs admission

2. What resuscitative measures are appropriate

3. Whether the patient needs urgent investigation /intervention

4. Where the patient is managed

Working Definitions and Level of Care

○ For practical purposes lower GI bleeding may be stratified into two broad groups

1. Minor bleeding

2. Significant bleeding

\section{Minor Bleeding}

Working definition of minor bleeding:

- Small volume bleed (few 'cupfulls', only on paper)

- Few episodes (<3 in 24 hours)

- No bleeding in last 6 hours

- No haemodynamic compromise (No tachycardia; No hypotension - overt or orthostatic)

- Proctosigmoidoscopy- a local anorectal self limiting cause is found or no active proximal bleeding.

- Not coagulopathic (INR/APTTR/ platelets - normal)

- Normal haemoglobin

- No major systemic co-morbidity

- Favourable social circumstances

- Patients satisfying all of these criteria for minor bleeding may not need admission as inpatients. However, this decision should be individualised and should be made by the middle grade surgeon on-Call. They will need to be reviewed in the outpatients clinic after appropriate investigations, such as a flexible sigmoidoscopy.

\section{Significant Bleeding}

\section{Working definition of significant bleeding}

- Suspected large volume bleeding based on history

- Any bleeding with haemodynamic compromise

- Any bleeding with Anaemia, thrombocytopenia or abnormal coagulation tests

- Any bleeding in patients with significant systemic comorbidity (such as cardiac failure/atrial fibrillation/Renal impairment/Cirrhosis/ COPD)

- Any bleeding in patients on therapeutic anticoagulation or are coagulopathic.

- Any bleeding in patients who are difficult to assess (such as patients with learning difficulties;patients under the influence of alcohol; confused patients with unclear history of events)

- Unfavourable social circumstances

- Bleeding in the elderly (age > 75 years)

- Patients with significant bleeding may further be stratified into three categories which will determine the pathway in the diagnostic and therapeutic algorithm [ Fig 1] 
Deeplaxmi Borle \& Senthil Kumar, "Practical Issues in the Decision Making and Management of Acute lower Gastrointestinal Bleeding: an Algorithmic Approach"

- Stable: No haemodynamic compromise or transient initial hypotension which responded well to fluid resuscitation and no or minimal ongoing bleeding and transfusion requirement not more than 2 units in 24 hours.

- Unstable: Intermittent hypotension associated with intermittent bleeding / Significant systemic comorbidity/ transfusion requirement 2-6 units in 24 hours.

- Exsanguinating bleeding: persistent hypotension or shock despite initial resuscitation and / or ongoing large volume bleeding. High transfusion requirements (> 6 units in 24 hours)

○ All patients with significant bleeding will need in patient care

- All patients with significant Lower GI bleed should be reviewed by a middle grade surgeon as soon as possible.

- All patients who are deemed unstable or have exsanguinating bleeding or who need urgent diagnostic or therapeutic intervention or who need HDU/ITU care should be discussed with the oncall consultant surgeon.

- Unstable patients should be considered for HDU/ITU care and there should be a low threshold to liaise with the critical care team during the initial assessment and resuscitation phase.

(B) Specific Clinical Management

The management of a patient with lower GI bleed may be divided into two distinct but related phases:

1. Initial evaluation and resuscitation and

2. Localisation and definitive treatment

Continual ongoing monitoring, planned periodical re-assessment; involving multidisciplinary teams when appropriate (haematologists, radiologists, anaesthetists, critical care team) and effective communication between and within teams especially at handover are important elements of care throughout both phases.

\section{Initial Evaluation and Resuscitation}

The emphasis during this initial phase is to start adequate resuscitative measures quickly and gather as much relevant clinical data simultaneously as resuscitation proceeds. An accurate diagnosis is not essential at this stage as the supportive measures are essentially generic for blood loss.

\section{Aspects of Resuscitation}

- Oxygen

○ High flow oxygen 10-15 litres / minute, ideally by a non-rebreathing mask should be administered.

- Adequate oxygenation in patients with GI bleed reduces the morbidity of interventions such as colonoscopy and angiography.

- IV Access And Fluids

- 2 large bore cannulae should be in place (at least 1 should be a 16 gauge). Seek the help of anaesthetists if there is a difficulty with iv access.

- Patients with severe bleeding and those with significant comorbidity should ideally have a central venous line inserted. These are also the patients who will need monitoring and resuscitation in a HDU environment. Discuss with critical care team.

- Blood

- Depending on the assessed severity of bleeding, the initial request for cross match should be for 4-6 units, in those with significant bleeding.

- If the patient has hypotension on admission, it is quite likely that there will be a need for transfusion.

- If there is exsanguinating bleeding, then type specific, uncrossmatched blood or $\mathrm{O}$ negative blood should be urgently requested.

- In a bleeding patient it is perhaps safe to aim for a haemoglobin of $10 \mathrm{gms} \%$ ( as opposed to a transfusion trigger of $7-8 \mathrm{gm} \%$ in 'subacute' presentations or chronic anaemia) 
Deeplaxmi Borle \& Senthil Kumar, "Practical Issues in the Decision Making and Management of Acute lower Gastrointestinal Bleeding: an Algorithmic Approach"

- If coagulopathy is present or likely, alert the transfusion service early for the potential need for blood products. The release of blood products will often need authorisation by the haematologist on-call with whom the case should be discussed.

\section{Correct Coagulopathy}

Coagulopathy may be the cause or effect of massive bleeding. It may exist at initial presentation or more commonly evolve during the course of the bleeding episode. It is vital to actively seek and manage the various derangements of the coagulation mechanism, initially and at planned intervals during the course of the bleeding episode.

○ If INR > 1.5 - discuss with haematologist. Will need FFP 10-15 mls $/ \mathrm{kg}$.

O If Platelets $<50,000$ - discuss with haematologist. Will need platelet transfusion in a bleeding patient, especially if any intervention such as angiography, colonoscopy or surgery is likely in the ensuing few hours.

- If on warfarin or known chronic liver disease- administer Vitamin K 10 mg slow iv. It usually takes about 6 hours to reverse warfarinisation.

- If on therapeutic heparin therapy- Stop heparin. May need protamine to reverse heparinisation depending on how dynamic the bleeding is and how unstable the patient is. Discuss with haematologist.

- If on Aspirin or Clopidogrel - withhold till the patient is stable and has stopped bleeding.

- If bleeding occurs in the ITU setting on a patient on Activated protein C therapy-Stop activated protein $\mathrm{C}$ and discuss with haematologist on call.

- Hypothermia makes coagulopathy worse and being proactive to achieve normothermia is essential.

- Maintain Normothermia

○ Infuse warmed iv fluids

- Blood and blood products should be given through a warmer whenever possible

- Active external forced air warming via a Bair-Hugger or similar device. If not practical, passive warming with blankets should be provided.

\section{HISTORY}

A comprehensive history should be elicited as, this will often guide or change management and help choose diagnostic and therapeutic modalities that are tailored to that patient.

In a patient who is critically ill or confused, the relatives, friends and carers are important sources of vital information. The patient's GP may be contactable and this avenue should be explored if necessary.

Request for the patients' old case notes should be made early (in A\&E), as this will often provide detailed additional information.

Age

The common causes vary depending on the age group. In adolescents and young adults the common causes of a significant lower GI bleed are Meckels' diverticulum and inflammatory bowel disease. In adults older than 70 years, diverticular disease, angiodysplasia and tumours are the common causes.

\section{History of Presenting Illness}

Important points in the history are

- Type of bleeding

- Bright red blood (with or without clots)

○ Dark blood ('maroon colored', 'altered')

- Black tarry (melena)

○ Was it mixed with stool? 
Deeplaxmi Borle \& Senthil Kumar, "Practical Issues in the Decision Making and Management of Acute lower Gastrointestinal Bleeding: an Algorithmic Approach"

- Frequency of bleeding

- Time of first bleeding

○ Intervals between bleeds

○ Number of bleeds

- Volume of bleeding

This is difficult to assess and is often an approximation. The aim is to differentiate between a large volume bleed and a relatively small amount of bleed. Using terms such as 'spoonfulls', 'cupfulls', 'pints' or ' bowlfulls' may help.

- Associated symptoms

- Episodes of dizziness or unconsciousness- suggest a large volume bleed

- Abdominal pain - may indicate diverticulitis or colitis

○ Anal symptoms - points to a local cause such as fissure or haemorrhoids

- Severe tenesmus - sign of proctitis.

- Any haematemesis or history of vomiting 'coffee grounds' - patients may be distracted or overwhelmed by rectal bleeding that they may not be forthcoming with a history of haematemesis.

- Trauma: a history of rectal trauma from insertion of foreign bodies may not be forthcoming. Gluteal stab wounds are notorious for associated rectal injury which can be subtle in its presentation.

- Recent polypectomy: delayed bleeding can occur up to 15 days after polypectomy (Waye 1996; Rex 1992).

\section{Past Medical History/ Co-Morbidity}

- Knowledge of co-morbidity and past medical history has, broadly, two important applications. Firstly, it allows interpretation of the patient's clinical picture in a more focussed perspective. This narrows the differential diagnosis and allows consideration of otherwise rare events if the clinical context is right (such as an aortoenteric fistula in a patient with a past history of prosthetic aortic graft). Secondly, this will provide an estimate of the physiological reserve of the patient and the potential risks, both of which will influence the choice of the choice of investigations, level of care and type of interventions.

- Case notes should be reviewed with special attention to endoscopy and colonoscopy reports, if any.

- History of diverticular disease, polyps, bowel cancer or inflammatory bowel disease provide vital clues to the probable diagnosis.

- History of cirrhosis or portal hypertension should alert to the possibility of esophageal or gastric variceal bleeding presenting as lower GI bleed. These patients also are prone for rectal varices (rare).

- Associated vascular disease (peripheral vascular disease, ischaemic heart disease, cerebrovascular disease): consider ischaemic bowel

- Atrial fibrillation: suspect ischaemic bowel.

- History of Aortic aneurysm surgery - suspect ischaemic colitis and aortoenteric fistula

- Aortic stenosis - associated with angiodysplasia.

- History of pelvic radiation for cervical, prostatic or rectal cancer: consider radiation proctitis. It may present from 9 months to 4 years after radiation therapy (Schultheiss 1997; Gilinsky 1983; Lucarotti 1991).

- In HIV positive patients: suspect CMV colitis, ulcers, Kaposis sarcoma and thrombocytopenia.

- In patients on chemotherapy- suspect neutropenic colitis.

- Note should be made of systemic co-morbidity of the patient. 
Deeplaxmi Borle \& Senthil Kumar, "Practical Issues in the Decision Making and Management of Acute lower Gastrointestinal Bleeding: an Algorithmic Approach"

- Cardiovascular- Ischaemic heart disease: Past or recent MI; Angina- stable/unstable; Cardiac failure; cardiomyopathy; Atrial fibrillation; Hypertension

○ Respiratory - COPD; Asthma

○ Chronic renal impairment/ failure

○ Cirrhosis

○ Diabetes

Drug History

- Aspirin, other NSAIDS and steroids increase the risk of lower GI bleed as well as upper GI bleed.

- Clopidogrel

- Warfarin

- Beta blockers - may not mount a tachycardic response to volume loss.

\section{EXAMINATION}

Meticulous examination and documentation of signs is essential to judge the impact of bleeding and the physiological reserve of the patient which will advise the diagnostic and therapeutic pathway chosen. Documentation is important for several reasons:

- Indicates a comprehensive assessment

- Trends in observations are important in assessing progress and response to an intervention and hence an initial comprehensive assessment establishes a baseline record.

- It is an important vehicle of communication between and within teams

- It has medicolegal significance.

The following points should be documented:

\section{General Examination}

Sensorium - drowsiness, confusion, anxiety? - May indicate cerebral hypoperfusion or sympathetic overactivity. The Glasgow coma scale or the AVPU system may be used.

Color (skin, conjunctiva, tongue, nails, palms) - pallor? Cyanosis?

Peripheries - cold and clammy?

Hydration - dry tongue? loss of skin turgor?

Heart rate and rhythm- Tachycardia? (beware of the patient on a beta blocker); Atrial fibrillation?.

Respiratory rate - Tachypnea like tachycardia is often an early sign in the face of hypovolemia and acidosis

Hypotension - Should be interpreted in the light of the patient's age

$\mathrm{SpO} 2$ - falling saturation $(<95 \%)$ may indicate ongoing bleeding, need for further resuscitation and possibly a need for escalation of the level of care (HDU/ITU)

Abdominal examination

Scars from previous surgery?

Skin bruising? (over warfarinisation/ heparin therapy/ thrombocytopenia)

Caput medusae?

Tenderness

Mass/fullness

Hepatosplenomegaly?

Free fluid? 
Deeplaxmi Borle \& Senthil Kumar, "Practical Issues in the Decision Making and Management of Acute lower Gastrointestinal Bleeding: an Algorithmic Approach"

Digital rectal examination - type of blood; clots? mass? fissure? foreign body?

\section{Localisation and Definitive Treatment}

The clinical context, history and examination usually provide enough clues to narrow down the working diagnosis to a few probable causes. As laid out in the algorithm, the severity of bleeding and available resources will dictate the pathway of management chosen [fig 1]. This however, needs to be individualised for the patient and the clinical context.

There are two adjuncts to the initial assessment that could be performed at the bedside - rigid proctosimoidoscopy and passage of a nasogastric tube.

\section{Proctosigmoidoscopy}

Rigid proctosigmoidoscopy is an important adjunct to clinical examination and should be performed in the intial 12-24 hours. The advantages of proctosimoidoscopy are:

- Identifies anorectal causes - hence helps plan the next appropriate intervention.

- Allows assessment of rectal mucosa and will aid in differentiating diffuse inflammation or ulceration (eg proctitis of ulcerative colitis, radiation proctitis) from focal lesions such as carcinoma.

- Allows tissue sampling - early diagnosis

- Allows local therapy - injection of haemorrhoids

- May be able to evacuate clots before colonoscopy improving the yield and success rate.

Nasogastric Tube

In patients presenting with severe haematochezia and hypotension, about 10-15\% will have an upper GI source (Jensen 1988). NG tube aspiration with or without lavage, in this group of patients, is a useful and easy bedside test. Aspiration of clear bile makes an upper GI source unlikely with the caveat that this can still be falsely negative in up to $10 \%$ especially if only clear fluid was aspirated (Cuellar 1990). Aspiration of blood or coffee grounds warrants urgent upper GI endoscopy.

\section{Colonoscopy}

Colonoscopy (or flexible sigmoidoscopy) should be the first choice investigation in stable patients and in selected unstable patients. The diagnostic accuracy of urgent colonoscopy is about 69\% (range from 40-90\%) in acute lower GI bleeding (Jensen 1988, Rossini 1989, Peura 1997, Zuckerman 1998). Early colonoscopy reduces hospital stay and costs (Strate 2003, Machicado 1997). Therapeutic colonoscopy also reduces the need for surgery (Jensen 2000).

Angiodysplasia are red fern like flat lesions ranging from 2-10 mm with or without a pale halo around. Although angiodysplasia may occur in the small bowel, they usually present as occult blood loss as opposed to the more acute and overt presentation of colonic angiodysplasia. Colonic angiodysplasia occur most frequently in the caecum (54\%), followed by the sigmoid colon (18\%) and the rectum (14\%) (Hochter 1985).

When a complete colonoscopy is performed with adequate views, the sensitivity for detecting angiodysplasia is more than $80 \%$ (Richter 1984, Clouse 1985, Moreto 1986, Hochter 1985). The use of narcotic analgesia and sedation reduces the sensitivity of colonoscopy in detecting angiodysplasia, by transiently reducing mucosal blood flow.

\section{Angiography and Angioembolisation}

- Angiography should be considered early in an unstable patient with ongoing bleeding and in a stable patient after failed colonoscopy and recurrent bleeding.

- It is sensitive for bleeding $>1 \mathrm{ml} / \mathrm{mt}$.

- Can identify multiple bleeding sites

- Angioembolisation can be performed

- May facilitate minimal access surgery (such as in Meckel's diverticulum) 
Deeplaxmi Borle \& Senthil Kumar, "Practical Issues in the Decision Making and Management of Acute lower Gastrointestinal Bleeding: an Algorithmic Approach"

- Can be repeated in patients with intermittent bleeding and can be performed intra-operatively if necessary.

- Intravascular vasopressin infusion may be used as a temporising measure

- If the patient subsequently needs surgery, segmental resection guided by pre-operative angiography results in significantly lower morbidity than in historic controls without localisation (Browder 1986; Drapanas 1973; Klas 1997; Nath 1981). This approach is also associated with lower rebleeding rates at one year (Parkes 1993).

- The complications of angiography/embolisation include anaphylaxis, renal toxicity and bowel ischemia. These risks need to be considered before embarking on this pathway and should be explained to the patients and relatives.

\section{Red Cell Scan}

Labelled red cell scan is sensitive for a rate of bleeding more than $0.1 \mathrm{ml} / \mathrm{mt}$ but specificity for site of bleeding is low. It helps to "screen out" non-significant bleeders, thereby help select patients for angiography and doubles the yield of angiography. Its limitations are availability and the inability to localise bleeding to a specific segment of the bowel. The advantages are that it is relatively noninvasive and the scans can be repeated in patients who bleed intermittently.

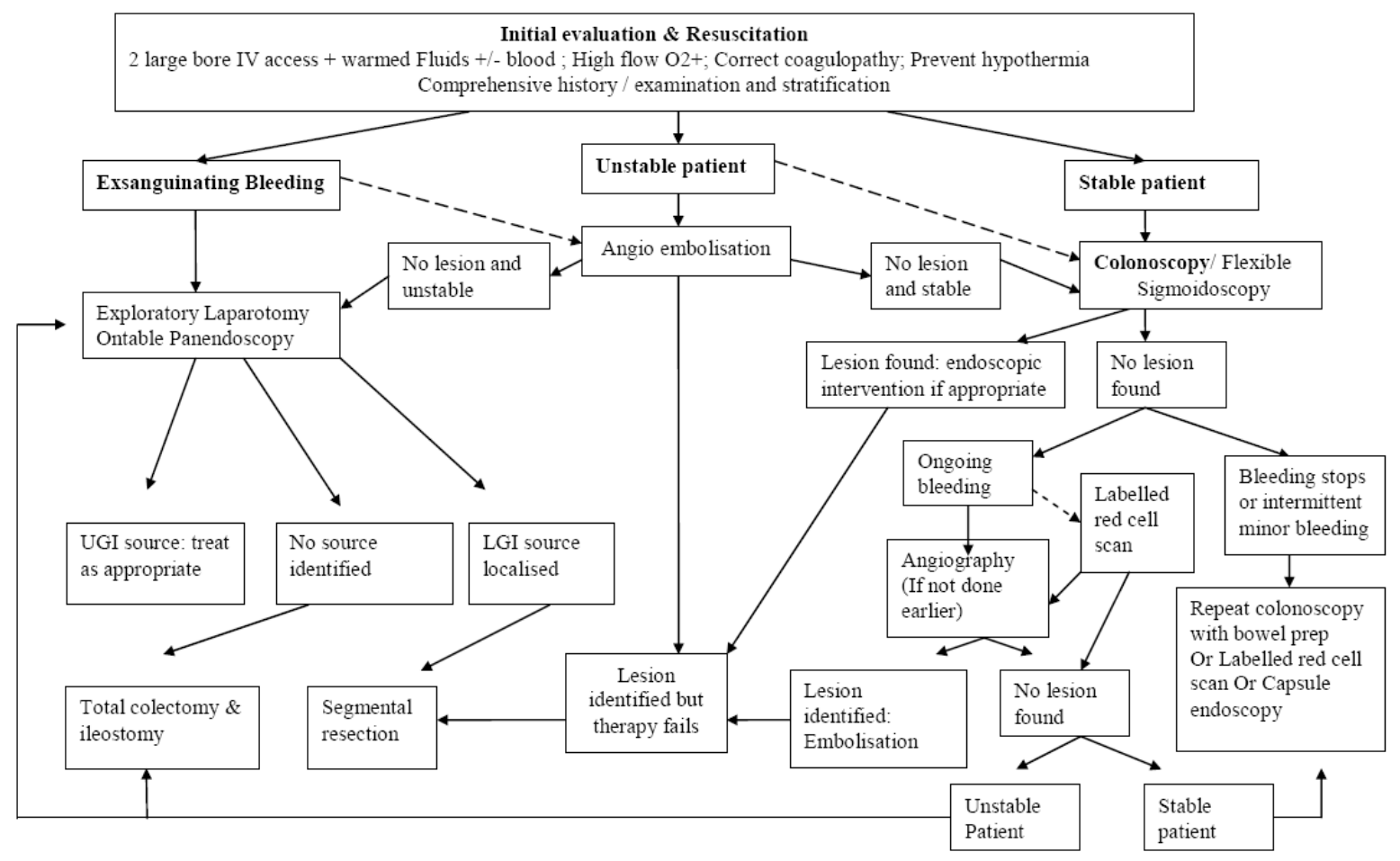

\section{Surgery}

Surgical intervention is required in $18-25 \%$ of patients who require a transfusion and surgery caries a mortality of 10\% (McGuire 1994, Bokhari 1996). Theatre, anaesthetists, ITU, endoscopist, and blood bank should be notified early. If the emergency theatre is occupied, then the need to open another theatre should be anticipated.

Directed segmental resection which has a better outcome than a blind total colectomy, should be aimed for. This may need on table panendoscopy if pre-operative methods had failed. The patient should be positioned accordingly. Abdomen may be prepared before induction of anaesthesia, so as not to save time to access.

Surgery in general is the last resort and is rarely necessary. However, it should be considered in patients with exsanguinating bleeding and in unstable patients when angiography or colonoscopy are not feasible or have failed due to technical or logistic reasons. The indications for surgery are (McGuire 1972, Newhall 1981, Milewski 1989, Field 1994, McGuire 1994).

- Persistent hypotension despite resuscitation 
Deeplaxmi Borle \& Senthil Kumar, "Practical Issues in the Decision Making and Management of Acute lower Gastrointestinal Bleeding: an Algorithmic Approach"

- Continued bleeding needing transfusion of a total of $>6$ units of packed cells and failure of localisation of bleeding despite panendoscopy, angiography and radionuclide imaging.

- Active bleeding from a segmental lesion amenable to cure or permanent haemostasis by surgery.

- Patient is an emergency surgical candidate with a reasonable life expectancy

\section{REFERENCES}

Bokhari M, Vernava AM, Ure T, Longo WE. Diverticular haemorrhage in the elderly - is it well tolerated? Dis Colon Rectum 1996; 39: 191-5.

Bramley PN, Masson JW, McKnight G, et al. The role of an open-access bleeding unit in the management of colonic haemorrhage. A 2-year prospective study. Scand J Gastroenterol 1996; 31: 764-9.

Browder W, Cerise EJ, Litwin MS. Impact of emergency angiography in massive lower gastrointestinal bleeding. Ann Surg 1986; 204: 530-6.

Clouse RE, Costigan DJ, Mills BA, Zuckerman GR. Angiodysplasia as a cause of upper gastrointestinal bleeding. Arch Intern Med 1985; 145: 458-61.

Cuellar RE, Gavaler JS, Alexander JA, et al. Gastrointestinal tract haemorrhage. The value of a nasogastric aspirate. Arch Intern Med 1990; 150: 1381-4.

Drapanas T, Pennington DG, Kappelman M, Lindsey ES. Emergency subtotal colectomy: preferred approach to management of massively bleeding diverticular disease. Ann Surg 1973; 177: 51926.

Farrell JJ, Friedman LS. The management of lower gastrointestinal bleeding. Aliment Pharmacol Ther 2005; 21: 1281-1298.

Field RJ Sr, Field RJ Jr, Shackleford S. Total abdominal colectomy for control of massive lower gastrointestinal bleeding. J Miss State Med Assoc 1994; 35: 29-33.

Gilinsky NH, Burns DG, Barbezat GO, Levin W, Myers HS, Marks IN. The natural history of radiation-induced proctosigmoiditis: an analysis of 88 patients. Q J Med1983; 52: 40-53.

Hochter W, Weingart J, Kuhner W, Frimberger E, Ottenjann R. Angiodysplasia in the colon and rectum. Endoscopic morphology, localisation and frequency. Endoscopy 1985; 17: 182-5.

Hoedema RE, Luchtefeld MA The management of lower gastrointestinal hemorrhage. Dis Colon Rectum 2005; 18 (11): 2010-24.

Jensen DM, Machicado GA, Jutabha R, Kovacs TO. Urgent colonoscopy for the diagnosis and treatment of severe diverticular disease. N Eng J Med 2000; 342: 78-82.

Jensen DM, Machicado GA. Diagnosis and treatment of severe haematochezia. The role of urgent colonoscopy after purge. Gastroenterology 1988; 95: 1569-74.

Klas JM RD. Surgical options in lower gastrointestinal bleeding. Semin Colon Rectal Surg 1997; 141: $478-81$.

Kollef MH, O'Brien JD, Zuckerman GR, Shannon W. BLEED: a classification tool to predict outcomes in patients with acute upper and lower gastrointestinal haemorrhage. Crit Care Med 1997; 25: 1125-32.

Lucarotti ME, Mountford RA, Bartolo DC. Surgical management of intestinal radiation injury. Dis Colon Rectum 1991; 34: 865-9.

Machicado GA, Jensen GM. Acute and chronic management of lower GI bleeding: cost effective approaches. Gastroenterologist 1997; 5:189-201.

McGuire HH Jr, Haynes BW Jr. Massive haemorrhage for diverticulosis of the colon: guidelines for therapy based on bleeding patterns observed in fifty cases. Ann Surg 1972;175: 847-55.

McGuire HH Jr. Bleeding colonic diverticula. A reappraisal of natural history and management. Ann Surg 1994; 220:653-6.

Milewski PJ, Schofield PF. Massive colonic haemorrhage -the case for right hemicolectomy. Ann R Coll Surg Engl 1989; 71: 253-9.

Moreto M, Figa M, Ojembarrena E, Zaballa M. Vascular malformations of the stomach and duodenum: an endoscopic classification. Endoscopy 1986; 18: 227-9. 
Deeplaxmi Borle \& Senthil Kumar, "Practical Issues in the Decision Making and Management of Acute lower Gastrointestinal Bleeding: an Algorithmic Approach"

Nath RL, Sequeira JC, Weitzman AF, Birkett DH, Williams LF Jr. Lower gastrointestinal bleeding. Diagnostic approach and management conclusions. Am J Surg 1981; 141: 478-81.

Newhall SC, Lucas CE, Ledgerwood AM. Diagnostic and therapeutic approach to colonic bleeding. Am Surg 1981;47: 136-42.

Parkes BM, Obeid FN, Sorensen VJ, Horst HM, Fath JJ. The management of massive lower gastrointestinal bleeding. Am Surg 1993; 59: 676-8.

Peura DA, Lanza FL, Gostout CJ, Foutch PG. The American College of Gastroenterology Bleeding Registry: preliminary findings. Am J Gastroenterol 1997; 92: 924-8.

Rex DK, Lewis BS, Waye JD. Colonoscopy and endoscopic therapy for delayed post-polypectomy haemorrhage. Gastrointest Endosc 1992; 38: 127-9.

Richter JM, Hedberg SE, Athanasoulis CA, Schapiro RH. Angiodysplasia. Clinical presentation and colonoscopic diagnosis. Dig Dis Sci 1984; 29: 481-5.

Rossini FP, Ferrari A, Spandre M, et al. Emergency colonoscopy. World J Surg 1989; 13: 190-2.

Schultheiss TE, Lee WR, Hunt MA, Hanlon AL, Peter RS, Hanks GE. Late GI and GU complications in the treatment of prostate cancer. Int J Radiat Oncol Biol Phys 1997; 37: 3-11.

Stratel L, Syngal S. Timing of colonoscopy: impact on length of hospital stay. Am J Gastroenterology. 2003; 98: 317-322.

Waye JD, Kahn O, Auerbach ME. Complications of colonoscopy and flexible sigmoidoscopy. Gastrointest Endosc Clin North Am 1996; 6: 343-77.

Zuckerman GR, Prakash C. Acute lower intestinal bleeding: part I: clinical presentation and diagnosis. Gastrointest Endosc 1998; 48: 606-17.

Citation: Deeplaxmi B, Senthil K. Practical Issues in the Decision Making and Management of Acute lower Gastrointestinal Bleeding: An Algorithmic Approach. International Journal of Research Studies in Medical and Health Sciences. 2017;2(2):4-13.

Copyright: () 2017 Deeplaxmi B, Senthil K. This is an open-access article distributed under the terms of the Creative Commons Attribution License, which permits unrestricted use, distribution, and reproduction in any medium, provided the original author and source are credited. 